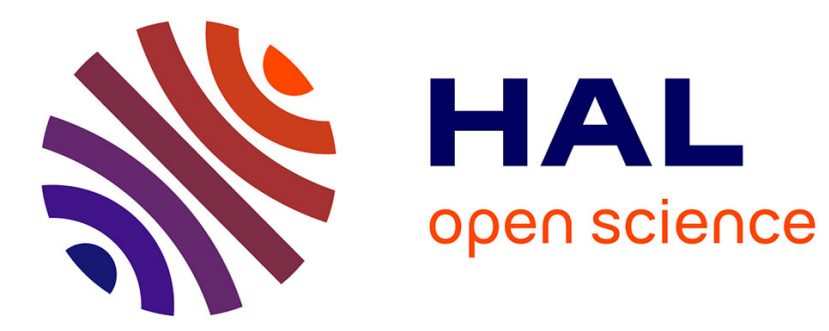

\title{
Energy Optimization based on the Redundancy in WSNs
}

\author{
Ibrahima Diané, Rahim Kacimi, Zoubir Mammeri, Ibrahima Niang
}

\section{To cite this version:}

Ibrahima Diané, Rahim Kacimi, Zoubir Mammeri, Ibrahima Niang. Energy Optimization based on the Redundancy in WSNs. 6th Joint IFIP Wireless and Mobile Networking Conference (WMNC 2013), Apr 2013, Dubaï, United Arab Emirates. pp. 1-7. hal-01240718

\section{HAL Id: hal-01240718 \\ https://hal.science/hal-01240718}

Submitted on 9 Dec 2015

HAL is a multi-disciplinary open access archive for the deposit and dissemination of scientific research documents, whether they are published or not. The documents may come from teaching and research institutions in France or abroad, or from public or private research centers.
L'archive ouverte pluridisciplinaire HAL, est destinée au dépôt et à la diffusion de documents scientifiques de niveau recherche, publiés ou non, émanant des établissements d'enseignement et de recherche français ou étrangers, des laboratoires publics ou privés. 


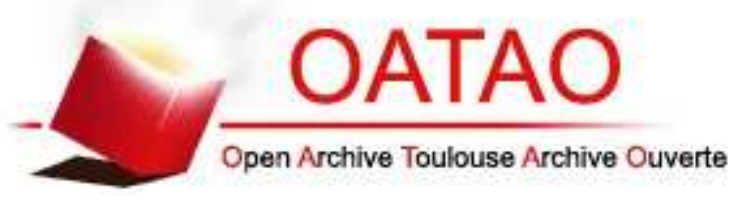

\section{Open Archive TOULOUSE Archive Ouverte (OATAO)}

OATAO is an open access repository that collects the work of Toulouse researchers and makes it freely available over the web where possible.

This is an author-deposited version published in : http://oatao.univ-toulouse.fr/ Eprints ID : 12443

The contribution was presented at WMNC 2013 : http://www.ieee.org/conferences_events/index.html

Official URL: http://dx.doi.org/10.1109/WMNC.2013.6548980

To cite this version : Diané, Ibrahima and Kacimi, Rahim and Mammeri, Zoubir and Niang, Ibrahima Energy Optimization based on the Redundancy in WSNs. (2013) In: 6th Joint IFIP Wireless and Mobile Networking Conference (WMNC 2013), 23 April 2013 - 25 April 2013 (Dubaï, United Arab Emirates).

Any correspondance concerning this service should be sent to the repository administrator: staff-oatao@listes-diff.inp-toulouse.fr 


\title{
Energy Optimization based on the Redundancy in WSNs
}

\author{
Ibrahima Diané ${ }^{1}$, Rahim Kacimi ${ }^{1}$, Zoubir Mammeri ${ }^{1}$, Ibrahima Niang ${ }^{2}$ \\ ${ }^{1}$ IRIT-UPS, University of Toulouse, France \\ \{diane, kacimi, mammeri\}@irit.fr \\ 2 LID-UCAD, University of Dakar, Senegal \\ iniang@ucad.sn
}

\begin{abstract}
Almost all WSNs (Wireless Sensor Networks) are deployed with some redundancy degree and redundancy is used only for robustness objectives. If not handled in an intelligent way, redundancy results in energy wasting because of (often unnecessary) redundant transmission and reception operations. We propose to take benefit from measurement redundancy to optimize the energy consumption and improve the end-to-end delay. We propose MR-LEACH (Measurement Redundancy aware $L E A C H$ ) protocol, which is an extension to the well-known LEACH protocol to improve energy consumption in cluster-based WSNs. In addition to cluster formation according to LEACH protocol redundant nodes are grouped taking into account their redundancy and only a single node transmits data in each redundant group. This technique significantly improves the energy consumption and ensures a better end-to-end delay. Through intensive simulations, we discuss the performance of our approach and show how it outperforms the original LEACH protocol in terms of network lifetime and end-to-end delay.
\end{abstract}

Index Terms-Wireless sensor networks, Clustering, measurement redundancy, geographical proximity

\section{INTRODUCTION}

A wireless sensor network can be defined as a set of interconnected sensor nodes, each sensor being provided with a transceiver. Sensor networks are a new generation of networks with specific properties. The miniaturization of sensors poses problems of communication and energy resources. It is necessary that the sensor node is smart enough to receive and to send data at low energy consumption with acceptable delay. The deployment of a sensor network raises problems of power management and end-to-end delay. Despite the use of clustering schemes, wireless sensor networks are still faced with energy consumption and delay issues. Most of existing work [1], [3], [5], [6], [8], [9], [10], [11], [13], [14], [15], [17], [19] propose improvements in terms of energy consumption. However, these solutions do not consider measurement redundancy. They typically use data aggregation and sleep periods to improve energy consumption and delay.

When the network size becomes large, its management raises difficulties. The structuring of a sensor network is one of the main mechanisms to save energy in each node, which allows to extend the system lifetime. One of the best known structures is the hierarchy. The hierarchization technique enables to partition the network into subsets in order to facilitate its management. In such a technique, network view becomes local; special nodes may have additional roles. The literature includes several contributions on hierarchization techniques of sensor networks, that is briefly presented in the following section. We distinguish two types of node groups: the zone and the cluster. A cluster is defined as a set of nodes with Cluster Head $(\mathrm{CH})$. The $\mathrm{CH}$ behaves as a relay between the sensor nodes and the base station directly or through other $\mathrm{CHs}$. $\mathrm{CH}$ usually has higher energy resources than other nodes in the network. $\mathrm{CH}$ is elected according to various criteria and information about the network: the level of energy, links with other sensor nodes, geographical position etc. A zone is defined as a set of nodes but does not have dedicated cluster head. Thus, a cluster is a subclass of a zone. Formation of groups (zones or clusters) is based on network information, thus requiring its instrumentation. These measures are taken in certain circumstances that may be static (such as node position in a fixed system) or dynamic (such as energy level of the nodes).

This article advocates the use of measurement redundancy for energy saving improvement. Indeed, we argue, in this paper, in favor of a grouping technique where the nodes having redundant measurements should be grouped. The measurement redundancy is the fact that several sensors in a same geographical proximity, report a same measurement value. Thus, the core idea of our approach is to determine and to group redundant nodes in order to have only one representing node by group which sends its data. Taking into account the measurement redundancy in clustered WSNs, our approach is based on a three-level hierarchical scheme as in LEACH [6].

The rest of this paper is organized as follows. Related work in the field of hierarchical power management in WSNs is summarized in section II. Section III describes MR-LEACH protocol and details the geographical proximity based grouping technique. In Section IV, we evaluate the performance of our approach. Finally, we conclude and present some research perspectives in Section V. 


\section{Related Work}

Low Energy Adaptive Clustering Hierarchy (LEACH), proposed by Heinzelman et al. [6], is a cluster-based approach in which clusters are formed in a self-organized way. Each cluster is controlled by a cluster head. With the aim of maximizing the network lifetime, the selection of the cluster head is based on the residual energy level of nodes. Cluster heads collect data from their cluster member nodes and, after processing, communicate the results to a global sink node. LEACH is a distributed clustering-based protocol that uses randomized rotation of the CHs to evenly distribute the energy load among the sensors in the network. LEACH assumes that the fixed sink is located far from the sensors and that all sensors in the network are homogeneous and battery-constrained. In order to reduce the amount of transmitted data to the sink, the CHs aggregate the collected data by their member nodes, and send an aggregated packet to the sink. However, although LEACH may increase the network lifetime, it has some limitations. LEACH assumes that all the nodes can transmit data with a high power to reach their $\mathrm{CH}$ and that each node has a computing power allowing it to support different MAC layers. Therefore, LEACH is not suitable for deployed networks in large regions. In addition, LEACH randomly selects a list of $\mathrm{CHs}$ and it poses no restrictions on their distribution. Thus, CHs may concentrate in one location and therefore there may be isolated nodes (without $\mathrm{CH}$ ). Moreover, in LEACH, the aggregation of data is centralized and is periodically executed by the $\mathrm{CH}$. This aggregation may also increase the end-to-end delay of transmitted packets if the redundancy management is not supported.

A centralized version of LEACH, LEACH-C, is proposed in [5], [4]. Unlike LEACH, where nodes self-configure themselves into clusters, LEACH-C utilizes the sink for cluster formation. During the setup phase of LEACH$\mathrm{C}$, the sink receives information regarding the location and energy level of each node in the network. Using this information, the sink finds a predetermined number of cluster heads and organizes the network into clusters. The cluster groupings are chosen to minimize the energy required for non-cluster-head nodes to transmit their data to their respective cluster heads. Although the other operations of LEACH-C are identical to those of LEACH, results presented in [5] indicate a definite improvement over LEACH. The authors in [5] provide two key reasons for the improvement:

- The sink utilizes its global knowledge of the network to produce better clusters that require less energy for data transmission.

- The number of cluster heads in each round of LEACH-C equals a predetermined optimal value, whereas for LEACH the number of cluster heads varies from round to round due to the lack of global coordination among nodes.
However, LEACH-C greatly increases the network overhead since all the sensors send their location information to the base station during each phase of $\mathrm{CHs}$ election. Moreover, the delay had not been improved. Several studies presented in the literature [16] showed that such centralized architecture does not support the scalability. Thus it is particularly suitable for small networks.

In [3], the authors proposed a hierarchical protocol based on LEACH. The CHs formed in LEACH are grouped and organized in a hierarchy. They showed that the energy consumption decreases when the number of levels of the hierarchy increases. However, they did not improve the end-to-end delay of LEACH.

Lindsey and Raghavendra [13] proposed Power-Efficient Gathering in Sensor Information Systems (PEGASIS), which avoids the assumption of direct communication and reduces the relatively large overhead of the LEACH protocol. In PEGASIS, the nodes form a chain, and each node stores in its routing table the addresses of upstream and downstream nodes. The data collection process is initiated at the far end of the chain. Each intermediate node aggregates the received data with its local data before transmitting the result to its upstream neighbor. The last node in the chain is responsible for transmitting to the sink node. In each round, a randomly chosen node from the chain transmits the aggregated data to the base station, thus reducing the per round energy expenditure compared to LEACH. However, the aggregation of data at all relay nodes results in a significant increase of end-toend delay of transmissions.

Hierarchical-PEGASIS [12], which is an extension to PEGASIS, is designed to address the delay incurred by packets during transmission to the base station. In order to improve the performance by reducing the delay in PEGASIS, messages are transmitted simultaneously. The effects of interference is reduced by carefully scheduling simultaneous transmissions. The approach taken by PEGASIS avoids the clustering overhead of LEACH, but still requires dynamic topology adjustment since sensor's energy is not tracked. Hence, every sensor must have the knowledge of its neighbor so that it knows where to route that data. So the topology needs adjustment and that can introduce significant overhead especially for networks with heavy traffic. Another similar protocol to PEGASIS called $C^{2} E^{2} S$, has been proposed in [7]. It is based on clusters and chains. It is a centralized protocol where the base station organizes the network on the basis of energy information of nodes.

Threshold sensitive Energy Efficient sensor Network protocol (TEEN) [14] is another hierarchical protocol coupled with data centric approach that is designed to react to sudden changes in the sensing attributes such as weather conditions. TEEN approach is more proactive rather than reactive. This is particularly important for time-sensitive applications. The sensor network architecture is based on a hierarchical grouping where closer nodes form clusters 
and this process goes on the second level until base station (sink) is reached. After the clusters are formed, the cluster head broadcasts the thresholds based on certain sensed attributes to the nodes. Once a node senses a value that exceeds the threshold value, it transmits data. However, the main drawback of TEEN is that, if the thresholds are not received, the nodes never communicate, and no data is transmitted to the user and the base station does not know the nodes that have exhausted their energy. Moreover, TEEN is not suitable for applications where periodic data are required since the user may not get any data at all if the thresholds are not reached.

The Adaptive Threshold sensitive Energy Efficient sensor Network protocol (APTEEN) [15] is an extension to TEEN and designed to capture both periodic data collections and reacting to time-critical events. The architecture is similar to TEEN. First the base station forms the clusters. Then the cluster heads broadcast the attributes, the threshold values, and the transmission schedule to all nodes. In addition, the cluster heads also perform data aggregation. Performance evaluation of TEEN and APTEEN has shown that they outperform LEACH. The main drawbacks are the overhead and complexity of forming clusters in multiple levels, implementing thresholdbased functions and dealing with attribute-based naming of queries.

In [2], [1], the authors proposed a clustering approach called Virtual Grid Architecture (VGA) to maximize the lifetime of wireless sensor networks where nodes are stationary or move at low speed. They used GPS-free approach [18] to build clusters. These clusters are fixed, disjoint and homogeneous in size with symmetrical shapes. VGA consists of a set of nodes, namely, Cluster Heads (CHs), that are elected periodically based on an eligibility criterion, which takes into account many changing parameters in the network. In VGA, the area of deployment of sensors is formed in a rectilinear virtual topology containing of small areas in the shape of a square, and in each of them, a node is selected as $\mathrm{CH}$. The data aggregation is performed at two levels: local and global. The local aggregation is performed by all CHs also called Local Aggregators (LAs), while the global aggregation is performed by a subset of LAs, called Master Aggregators (MAs). However, the determination of all MAs is a hard problem. The heuristics that have been proposed to form the set MAs from the set LAs, had as objective the maximization of the lifetime of sensor networks.

Younis et al. [19] have proposed HEED, whose main goal is to form efficient clusters for maximizing network lifetime. The main assumption in HEED is the availability of multiple power levels at sensor nodes. Unlike previous techniques, HEED makes no restriction on the distribution and the density of nodes. It depends neither on the topology of the network nor of its size, but it assumes that the sensors are able to change their transmission power. HEED is a distributed clustering protocol that periodically selects cluster heads according to a hybrid function between their residual energy and a secondary parameter, such as node proximity to its neighbors or node degree. The cost of a cluster head is defined as its average minimum reachability power (AMRP). AMRP is the average of the minimum power levels required by all nodes within the cluster range to reach the cluster head. AMRP provides an estimate of the communication cost. At every iteration of HEED, each node which has not selected a cluster head sets its probability $\mathrm{PCH}$ of becoming the cluster head. This probability depends on its residual energy, the global energy in the network and the optimal number of clusters. However, the evaluation of the global energy raises a challenge because of the absence of any central control. Another problem lies in determining the optimal number of clusters. In addition, HEED does not specify any particular protocol to use for communication between the CHs and the sink.

The authors in [9], [10] proposed a self-organisation technique based on the clustering approach to optimize the energy consumption in sensor networks. This technique consists in grouping the nodes close geographically in clusters. It requires determining parameters to produce a small number of clusters, homogeneous clusters (in size and radius), and stable clusters. In addition, the size of the generated clusters is between two thresholds ThreshLower and ThreshUpper, which respectively represent the minimum and maximum number of sensors in a cluster. These two thresholds are chosen arbitrarily or depend on the network topology. The process of $\mathrm{CH}$ election is periodic after the lapse of a certain period in order to equitably distribute the energy consumption among the sensors during the network lifetime. In their technique, sensors are assumed to have topological knowledge at two hops, they can change their transmission power, and operate in an asynchronous manner without central control. In the purpose to generate balanced clusters, [9] restricts both diameter and size of the cluster. Two thresholds are used to control the size of generated clusters and the distances between cluster members and their corresponding clusterhead are at most two hops. However, no solutions were provided for thresholds settings and intercluster communication.

\section{REDUNDANCY IN WIRELESS SENSOR NETWORKS}

\section{A. Problem statement}

Despite the use of clustering techniques, WSNs are still faced with problems of energy consumption and delay. Most of the work [1], [3], [5], [6], [8], [9], [10], [11], [13], [14], [15], [17], [19] propose improvements regarding energy consumption without taking into account the measurement redundancy which is also a source of unnecessary energy consumption. The initial placement description may explicitly specify some redundancy in the deployed sensor nodes. Wireless sensor networks are characterized by a large number of nodes. The measurement redundancy 


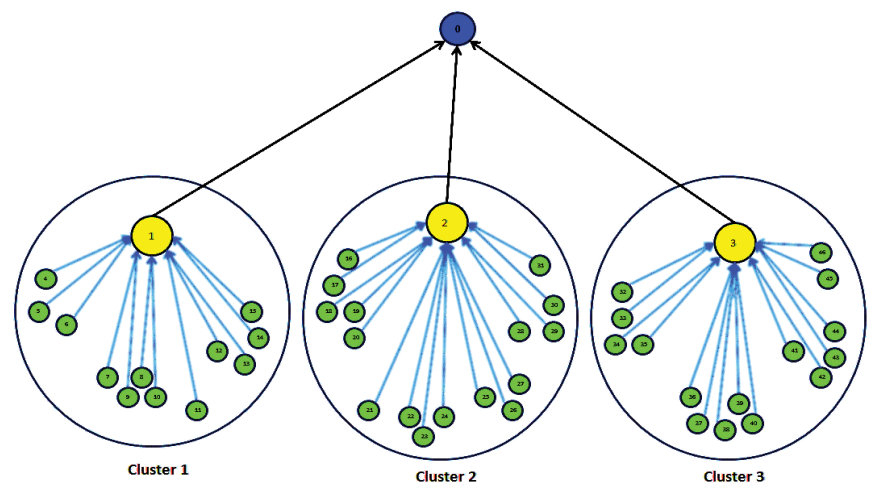

Fig. 1. A three-level hierarchical sensor network

may result from this high density of nodes. Multiple neighboring nodes, which are placed at specific locations and/or at given distance each from other, may detect the same event and/or report the same periodic value. It is therefore necessary to include mechanisms to support possible measurement redundancy. Therefore, the issue is the focus on measurement redundancy in wireless sensor networks for energy consumption improvement.

This article proposes and analyzes a technique which takes account of the measurement redundancy in hierarchical wireless sensor networks.

\section{B. Grouping technique by geographical proximity}

Clustered architectures are widely used in WSNs for their scalability. They allow a more efficient use of limited resources such as bandwidth and energy.

MR-LEACH uses a grouping technique based on geographic proximity. Depending on the geographic location, our technique allows the grouping of the non-cluster-head nodes having the same measurement values. Thus, only the group representing nodes send their packets. This way, the intra-cluster communications are reduced and the energy consumption of the nodes is optimized.

1) Assumptions: For easier understanding of our scheme in the remainder of this paper, we make some reasonable assumptions.

- As LEACH, a three-level network architecture is established.

- Nodes are densely and randomly deployed on a square area and their placement is known.

- Nodes observe the same physical phenomenon, generate a periodic monitoring traffic and send it to the BS through the cluster-heads. Additional changes are possible to support sporadic traffic such as alarms.

- No information on a possible redundancy is explicitly provided.

Figure 1 shows the basic architecture of our solution.

2) Principe of our approach: MR-LEACH combines geographical proximity of the nodes and their readings redundancy in order to reduce the number of the intracluster transmissions.

The initialization of MR-LEACH protocol comprises two main stages: In the first stage, the $\mathrm{CHs}$ collect and observe the cluster readings, while in the second they determine the measurement redundancy and group the redundant nodes. We give more details hereafter.

- Observation stage: as soon as the sensor nodes are deployed on the network, the clusters are formed and each $\mathrm{CH}$ collects the readings of the nodes. This phase is dimensioned by the duration IntMeasure.

- Grouping stage: after a sufficiently observation time (IntMeasure), the $\mathrm{CHs}$ have a good knowledge of their cluster readings. Thereafter, they analyze the collected measurements then based on the geographic proximity of the nodes they determine the groups of redundant nodes.

After identifying the redundancy groups, only one representing node will send its readings and the other nodes stop their transmissions.

As depicted by Fig. 2, at the end of the initialization phase, MR-LEACH works by periodic superframes. The superframes are divided into a set of time slots which are allocated to the representing nodes to send their data to the $\mathrm{CH}$. Other time slots are assigned to the $\mathrm{CHs}$ to send the data to sink. After each superframe, the role of the representing node rotates between the nodes of the group. A set of superframes forms a round and MR-LEACH ensures that in each round all nodes send their readings at least once in turn.

Geographical proximity calculation method: The geographical proximity exists if the distance between the nodes is less than the given threshold $d$. This proximity is deduced directly from the Euclidean distance between nodes.

Let $x_{i}$ and $y_{i}$ represent the coordinates of a $i^{\text {th }}$ node, then $i^{\text {th }}$ node is like a point $N_{i}$ in the space that can be represented as $N_{i}=\left(x_{i}, y_{i}\right)$. Euclidean distance between two nodes $N_{i}$ and $N_{j}$ is by definition:

$$
d_{2}\left(N_{i}, N_{j}\right)=\sqrt{\left(x_{i}-x_{j}\right)^{2}+\left(y_{i}-y_{j}\right)^{2}}
$$

For that there is geographical proximity between a set of nodes, it is necessary that:

$$
d_{2}\left(N_{i}, N_{j}\right) \leq d+\varepsilon
$$

Geographical proximity is only checked between sensor nodes which report the same measure during the time interval IntMesure. It is therefore checked between the sensor nodes which are likely to be redundant. Figure 3 shows the architecture of network after applying our grouping technique. A description of our technique is given below.

During the observation phase of measurements (IntMeasure), each $\mathrm{CH}$ creates a table of values (ValTable) where it will store the reported measurement 


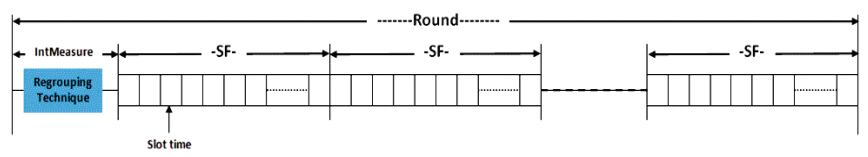

Fig. 2. Representation of solution round

values by the sensor nodes members of the cluster. This table will be used thereafter to calculate the redundancy matrix (reGroup) as detailed in Algorithm 1. Each $\mathrm{CH}$ then verifies the geographical proximity of all sensor nodes that reported the same measurement value (with some precision $\alpha$ ) during IntMeasure. Each $\mathrm{CH}$ then places in the same row of the matrix reGroup, the identifiers of all sensor nodes which are in the same geographical proximity. After this geographical proximity grouping, each $\mathrm{CH}$ asks some sensor nodes to fall asleep for the next super-frame so that only one node in each group at a time (or at the same time), during the next superframe.

This process is repeated until the end of the round which corresponds to clusters lifetime as shows in the figure 2 . There will be again clusters formation and application of grouping technique by geographical proximity.

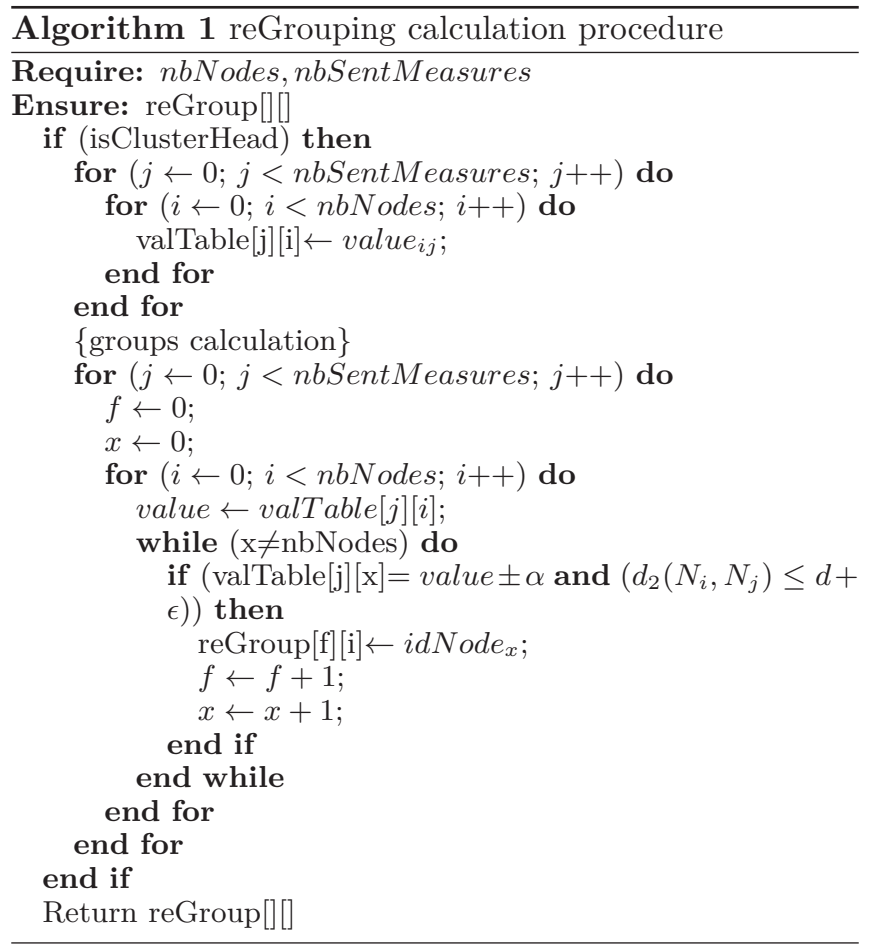

\section{Performance Evaluation}

To evaluate the performance of MR-LEACH, we simulated it using the NS2 simulation tool which provides an opportunity to integrate the LEACH protocol. Firstly, we integrated the implementation of LEACH. Then, to

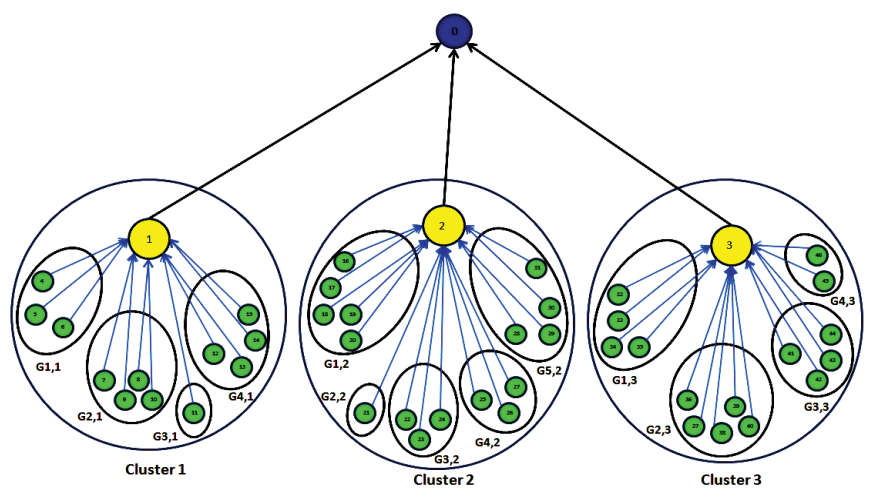

Fig. 3. Sensor Network after geographical proximity grouping

integrate our grouping technique by geographical proximity, we modified LEACH according to the hierarchical structure in the association commands of the MAC layer and the method of filling the redundancy table at the physical layer.

We compared our design with LEACH protocol. For this, we performed simulations with the same parameters and metrics for the two approaches. We are interested primarily in the energy consumption of nodes, which represents an important criterion for determining the lifetime of a sensor network. We evaluated the end-to-end delay as it is a performance criteria for many monitoring applications over WSN.

\section{A. Simulation set-up}

To investigate the behavior of MR-LEACH we used a network scenario consisting of 100 to 1000 nodes randomly deployed over a $250 * 250 \mathrm{~m}^{2}$ area. The coordinates of the sink node are $(135,155)$. The nodes know their position and can communicate it to their respective $\mathrm{CH}$.

TABLE I

SimUlation PARAMETERS

\begin{tabular}{|r|c|}
\hline Simulation area & $250 * 250 \mathrm{~m}^{2}$ \\
\hline number of nodes & {$[100,1000]$} \\
\hline Packet length & 55 bytes \\
\hline Initial node energy & $2 \mathrm{j}$ \\
\hline Transmission bit rate & $250 \mathrm{kbs}^{-1}$ \\
\hline Transmit power & $24.75 \mathrm{~mW}$ \\
\hline Receive power & $13.5 \mathrm{~mW}$ \\
\hline Idle power & $6.5 \mathrm{~mW}$ \\
\hline Sleep power & $15 \mu \mathrm{W}$ \\
\hline
\end{tabular}

Under all these simulation settings and for each given network size, we collected and averaged the results of 10 runs.

\section{B. Performace metrics}

The performance metrics in our study are as follows:

1) Energy consumption: To evaluate the energy consumption, we recovered the energy levels at the start 


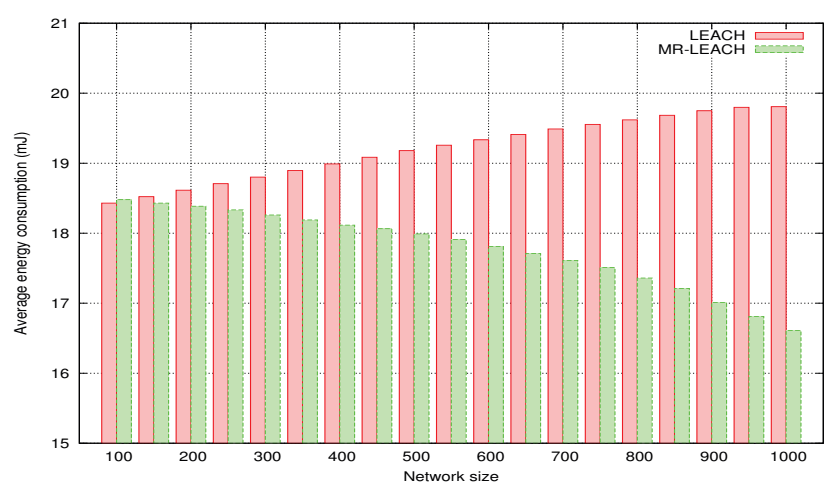

Fig. 4. Average energy consumption of each $\mathrm{CH}$ during one round

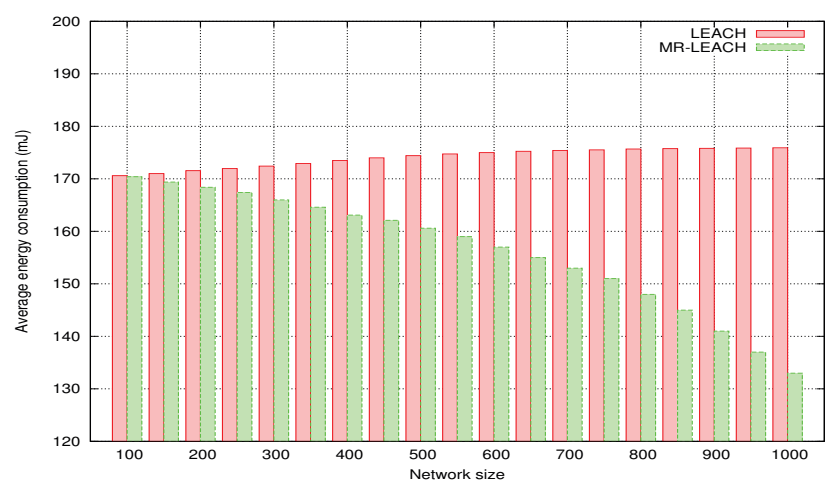

Fig. 5. Average energy consumption of each node after 20 rounds

and the current energy levels of sensor nodes from trace files generated by simulations.

2) Network lifetime: The network lifetime is defined as the estimated time before the network becomes nonfunctional. To evaluate the network lifetime, we recovered from the trace files generated by the simulations, the time after which the energy level of the nodes is zero. Energy levels are recovered after each round.

3) End-to-end Delay: To evaluate the end-to-end delay, we recovered the time of collection and receiving packets from the trace files generated by simulations.

Results obtained for metrics of interest are shown in figures 4, 5, 6 and 7 (with $5 \%$ of the nodes being cluster-heads).

\section{Comparison}

Fig. 4 shows the energy consumption of the clusterheads versus the number of nodes in the network. From the figure we can see that when the network size increases the energy consumption of the CHs in MR-LEACH decreases, in contrast of LEACH. Indeed, with high density the measurement redundancy increases. Thus, the $\mathrm{CHs}$ receive less data. This is because, the number of groups increases which reduces the number of transmissions in the

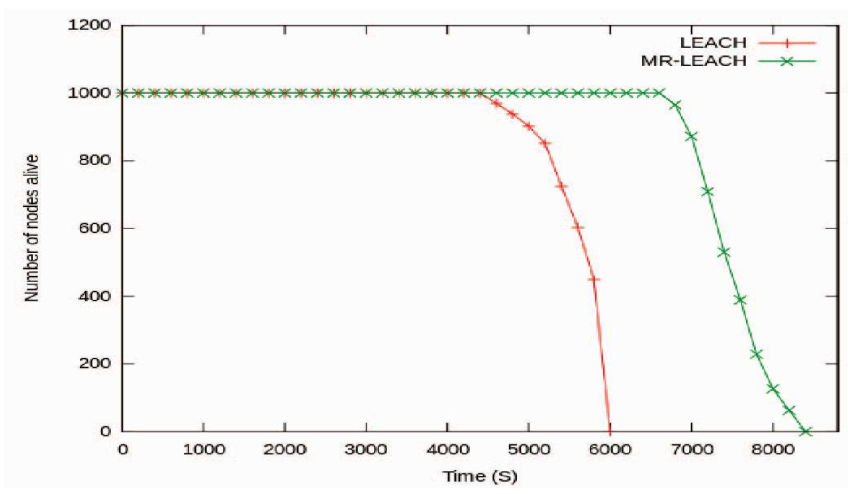

Fig. 6. Network lifetime

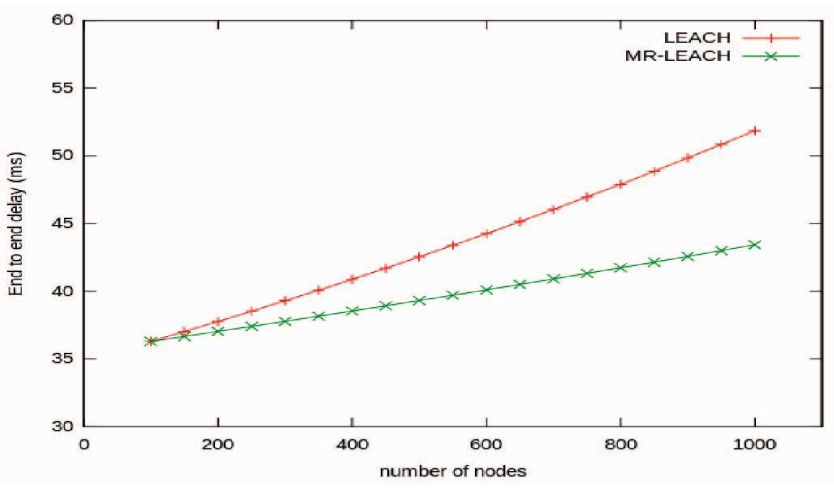

Fig. 7. End-to-end delay

clusters. Furthermore, the curves of Fig. 4 also show that the performance gap between MR-LEACH and LEACH becomes more significant when the network size increases.

Fig. 5 compares the average energy consumption of the nodes. We run the simulations during 20 rounds, each round corresponds to 25 seconds. The reason behind the 20 rounds is to allow all nodes to behave as a $\mathrm{CH}$ at least once. We note that in MR-LEACH, energy consumption of nodes decreases when the number of nodes increases, contrary to LEACH. Indeed, with a high density, a node communicates less with its $\mathrm{CH}$. Thus, the $\mathrm{CHs}$ receive less packets due to the measurement redundancy management. So, when the number of nodes is being higher, they are grouped and only the group representing nodes send their packets in the clusters.

Fig. 6 shows how the network lifetime is affected over time using MR-LEACH versus LEACH. In this set of experiments, we consider a network size of 1000 sensor nodes. Figure 6 shows that MR-LEACH has a greater network lifetime compared with LEACH. We found that MR-LEACH is more effective to extending the network lifetime. Indeed, compared to LEACH, it takes approximately 1.5 times longer until the first node dies and approximately 1.4 times longer until the last node dies. 
Moreover, this gain in term of network lifetime can be easily inferred from the results of Fig. 5 on the energy consumption.

We now discuss the impact of different network sizes on the average end-to-end delay. Fig. 7 plots the results of the average end-to-end delay evolution. As first result, we can see that the average delay grows with increasing of the network size. However, this increasing is less significant for MR-LEACH due to measurement redundancy consideration. In fact, the $\mathrm{CH}$ in MR-LEACH expects a number of packets $k \ll n$ before the aggregation, where $n$ is the size of the cluster.

\section{Conclusion}

This paper addressed the energy efficiency in the clustered wireless sensor networks. Our solution consists in taking benefit from the measurement redundancy in such networks. In this context, there is need of approaches which can manage these WSNs in a better way. We proposed MR-LEACH (Measurement Redundancy aware LEACH) protocol, which is an extension to the wellknown LEACH protocol to improve energy consumption in cluster-based WSNs. MR-LEACH has several strengths. It combines the geographical proximity of the nodes with the measurement redundancy in order to improve the energy efficiency and provides better end-to-end communication delays. The performance of our solution has been investigated through intensive simulations. Furthermore, we assessed how it outperforms LEACH protocol by extending the network lifetime and bounding the end-to-end communication delay. In our future work, we would like to extend our approach to consider event-triggering WSN and initial WSN deployment including explicit redundancy specification.

\section{REFERENCES}

[1] J.N. Al-Karaki, R. Ul-Mustafa, and A.E. Kamal. Data aggregation in wireless sensor networks - exact and approximate algorithms. In High Performance Switching and Routing, 2004. HPSR. 2004 Workshop on, pages $241-245,2004$.

[2] J.N. Al-Karaki, R. Ul-Mustafa, and A.E. Kamal. Data aggregation and routing in wireless sensor networks: Optimal and heuristic algorithms. Computer Networks, 53(7):945 - 960, 2009.

[3] S. Bandyopadhyay and E.J. Coyle. An energy efficient hierarchical clustering algorithm for wireless sensor networks. In INFOCOM 2003. Twenty-Second Annual Joint Conference of the IEEE Computer and Communications. IEEE Societies, volume 3, pages 1713 - 1723 vol.3, march-3 april 2003.

[4] V. Geetha, P.V. Kallapur, and S. Tellajeera. Clustering in wireless sensor networks: Performance comparison of leach \& leach-c protocols using ns2. Procedia Technology, 4:163-170, 2012 .

[5] W.B. Heinzelman, A.P. Chandrakasan, and H. Balakrishnan. An application-specific protocol architecture for wireless microsensor networks. Wireless Communications, IEEE Transactions on, 1(4):660-670, October 2002.

[6] W.R. Heinzelman, A. Chandrakasan, and H. Balakrishnan. Energy-efficient communication protocol for wireless microsensor networks. In System Sciences, 2000. Proceedings of the 33rd Annual Hawaii International Conference on, page $10 \mathrm{pp}$. vol.2, jan. 2000 .
[7] T.T. Huynh and C.S. Hong. An energy* delay efficient multihop routing scheme for wireless sensor networks. IEICE transactions on information and systems, 89(5):1654-1661, 2006.

[8] N. Kumar and J. Kaur. Improved leach protocol for wireless sensor networks. In Wireless Communications, Networking and Mobile Computing (WiCOM), 2011 7th International Conference on, pages 1-5. IEEE, 2011.

[9] M. Lehsaini, H. Guyennet, and M. Feham. A novel clusterbased self-organization algorithm for wireless sensor networks. In Collaborative Technologies and Systems, 2008. CTS 2008. International Symposium on, pages 19 -26, may 2008.

[10] M. Lehsaini, H. Guyennet, and M. Feham. An efficient clusterbased self-organisation algorithm for wireless sensor networks. International Journal of Sensor Networks, volume 7:85-94, March. 2010.

[11] Y. Li, N. Yu, W. Zhang, W. Zhao, X. You, and M. Daneshmand. Enhancing the performance of leach protocol in wireless sensor networks. In Computer Communications Workshops (INFOCOM WKSHPS), 2011 IEEE Conference on, pages 223-228, 2011.

[12] S. Lindsey, C. S. Raghavendra, and M. Sivalingam, Krishna. Data gathering in sensor networks using the energy delay metric. In Proceedings of the 15th International Parallel $\&$ Distributed Processing Symposium, IPDPS '01, pages 188-, Washington, DC, USA, 2001. IEEE Computer Society.

[13] S. Lindsey and C.S. Raghavendra. Pegasis: Power-efficient gathering in sensor information systems. In Aerospace Conference Proceedings, 2002. IEEE, volume 3, pages 3-1125 - 3-1130 vol.3, 2002.

[14] A. Manjeshwar and D.P. Agrawal. Teen: a routing protocol for enhanced efficiency in wireless sensor networks. In Parallel and Distributed Processing Symposium., Proceedings 15th International, pages $2009-2015$, april 2001.

[15] A. Manjeshwar and D.P. Agrawal. Apteen: a hybrid protocol for efficient routing and comprehensive information retrieval in wireless sensor networks. In Parallel and Distributed Processing Symposium., Proceedings International, IPDPS 2002, Abstracts and $C D-R O M$, pages $195-202,2002$.

[16] M. Perillo and W. Heinzelman. Wireless sensor network protocols. Algorithms and Protocols for Wireless and Mobile Networks, Eds. A. Boukerche et al., CRC Hall Publishers, 2004.

[17] G. Ran, H. Zhang, and S. Gong. Improving on leach protocol of wireless sensor networks using fuzzy logic. Journal of Information \& Computational Science, 7(3):767-775, 2010.

[18] A. Savvides, C.C. Han, and M.B. Strivastava. Dynamic finegrained localization in ad-hoc networks of sensors. In International Conference on Mobile Computing and Networking: Proceedings of the 7 th annual international conference on Mobile computing and networking, volume 2001, pages 166-179, 2001.

[19] O. Younis and S. Fahmy. Heed: a hybrid, energy-efficient, distributed clustering approach for ad hoc sensor networks. Mobile Computing, IEEE Transactions on, 3(4):366 - 379, oct.dec. 2004. 